\title{
Review Article \\ Review on Recent Developments in Laser Driven Inertial Fusion
}

\author{
M. Ghoranneviss and A. Salar Elahi \\ Plasma Physics Research Center, Tehran Science and Research Branch, Islamic Azad University, Tehran, Iran \\ Correspondence should be addressed to A. Salar Elahi; salari_phy@yahoo.com
}

Received 25 February 2014; Accepted 1 March 2014; Published 7 April 2014

Academic Editor: Hamid Rouhparvar

Copyright (C) 2014 M. Ghoranneviss and A. S. Elahi. This is an open access article distributed under the Creative Commons Attribution License, which permits unrestricted use, distribution, and reproduction in any medium, provided the original work is properly cited.

\begin{abstract}
Discovery of the laser in 1960 hopes were based on using its very high energy concentration within very short pulses of time and very small volumes for energy generation from nuclear fusion as "Inertial Fusion Energy" (IFE), parallel to the efforts to produce energy from "Magnetic Confinement Fusion" (MCF), by burning deuterium-tritium (DT) in high temperature plasmas to helium. Over the years the fusion gain was increased by a number of magnitudes and has reached nearly break-even after numerous difficulties in physics and technology had been solved. After briefly summarizing laser driven IFE, we report how the recently developed lasers with pulses of petawatt power and picosecond duration may open new alternatives for IFE with the goal to possibly ignite solid or low compressed DT fuel thereby creating a simplified reactor scheme. Ultrahigh acceleration of plasma blocks after irradiation of picosecond (PS) laser pulses of around terawatt (TW) power in the range of $10^{20} \mathrm{~cm} / \mathrm{s}^{2}$ was discovered by Sauerbrey (1996) as measured by Doppler effect where the laser intensity was up to about $10^{18} \mathrm{~W} / \mathrm{cm}^{2}$. This is several orders of magnitude higher than acceleration by irradiation based on thermal interaction of lasers has produced.
\end{abstract}

\section{Introduction}

Controlled generation of fusion energy for power stations received an essential turning point by interaction of picosecond laser pulses of powers above terawatts with plasmas resulted in ultrahigh acceleration of plasma layers with a thickness of dielectric increased skin depths [1]. These plasma blocks contained directed energetic ions with extremely high ion current densities opening a new way of ignition of fusion by direct laser generation of a fusion flame in uncompressed solid fuel [2]. This reaction as a kind of side-on ignition was considered before as the following described hydrodynamic Chu-model but only the new experimental results of the plasma blocks provided the necessary conditions for a plane geometry interaction for fusion of deuterium-tritium. Fast ignition was the alternative first formulated by Tabak et al. [3] and elaborated [4] following the discussions about the highest deuterium-tritium (DT) fusion gains based on shockfree spherical ideal compression at thermal ignition [5-7]. The gains of more than $10^{12}$ fusion neutrons were significantly higher than all earlier attempts with exploding pushers
[8], where shocks led to strong degrading deviations from the ideal adiabatic implosion. The ideal adiabatic compression led to the highest gains at volume burn and the discovery of volume ignition [9], confirmed as "Wheeler modes" [10] and then summarized [8]. The discovery of this shockfree compression was reached systematically by varying the experimental parameters in parallel to computations, which, in contrast to those of exploding pushers, arrived at the highest gains as predicted [9], and the result [5] of which was called "Yamanka compression." Gains above $10^{13}$ neutrons were achieved [11] and as well as compression to 2000 times that of the solid state, where it was crucial that laser beam smoothing [12] be used [13]. Further improvement to gains led to gains of $2 \times 10^{14}$ neutrons [14] all with direct drive and volume ignition [8] in contrast to about 1000 times lower gains with indirect drive spark ignition [15]. What was most disappointing was the rather low maximum temperature $T$ at the highest compression [11] of only about $=3 \times 10^{6} \mathrm{~K}$, while the theory of spherical compression and thermal ignition said it should have reached higher temperatures. Nevertheless, the spherical compression and thermal ignition scheme was 
followed up by building the $\$ 4$ Billion laser facility NIF (National Ignition facility) under the initial leadership of Campbell (2006) followed by Moses et al. (2006) leading close to the historic first controlled exothermic nuclear fusion reactions [16], while simultaneously it was considered by Tabak et al. [3] to overcome the problem of the unexpected low temperature at compressions [11] by "fast ignition." The scheme at the first discussions (2006) was to use the spherical plasma compression to more than 1000 times the solid state density and to produce an additional heating of the center area by an additional laser pulse of picoseconds duration with an energy in the range above $10^{5} \mathrm{~J}$. In order to produce laser pulses of this short duration and power above terawatt (TW) and up to more than petawatt (PW), the chirped pulse amplification CPA $[17,18]$ was invented for solid state lasers. Another method is the dye laser pulse amplification by $\mathrm{KrF}$ lasers known as the Szatmari-Schäfer method [19, 20]. Since the controlled generation of clean, safe, virtually inexhaustive, and everywhere accessible fusion energy at sufficiently low cost in power stations using nuclear fusion reactions is an extremely important goal to overcome the problems inherent in the present energy sources, an enormous budget has been and will further be spent into research to solve this problem. A summary of this work can be found in a special issue of the IAEA based journal "Nuclear Fusion" which shows how many of the new physics problems have been solved and break-even has almost been reached. The main effort has been devoted to the reaction of DT at temperatures between 10 and 100 Million degrees centigrade where the reacting fuel, a high temperature plasma, has to be confined by magnetic fields (MCF). A review of the forthcoming ITER project and the complementary stellarator program is given in this volume by G. Janeschitz and R. Wolf. So far, the highest fusion gain has been achieved in JET (Joint European Torus) where a magnetically confined plasma torus (D:T ratio of $40: 60$ ), heated by $21 \mathrm{MW}$ of neutral beam and $3 \mathrm{MW}$ of electromagnetic wave power, has, for 2 seconds, produced $16 \mathrm{MW}$ of fusion power representing a fusion gain of $66 \%$ $[21,22]$. The extensive work on IFE and its success, with fusion gains not far below break-even, is traditionally based on lasers with pulses in the ns range and, for the last few years, on the new direction of fast igniters with PS laser pulses as reviewed in the following Section 2. These broad stream IFE developments are modified by a few new experiments which arrived at results significantly different form the broad stream observations. These anomalous results were reported by Sauerbrey, by Jie Zhang et al, and by Badziak et al. and could be understood only by realizing that theses authors used laser pulses of very high quality with suppression of prepulses. The main motivation for this explanation was guided by the knowledge of earlier computations shown in the following Figure 1. A consequence is that a modification of the fast ignition is possible, which may lead to a very simplified and therefore lower cost fusion reactor using plasma blocks with extremely high ion beam current densities. More details of these new aspects are described in Section 3. Sauerbrey's experimental discovery of ultrahigh laser acceleration of plasma by nonlinear forces will be presented in section 4 . Analysis of acceleration measurements with doppler effect

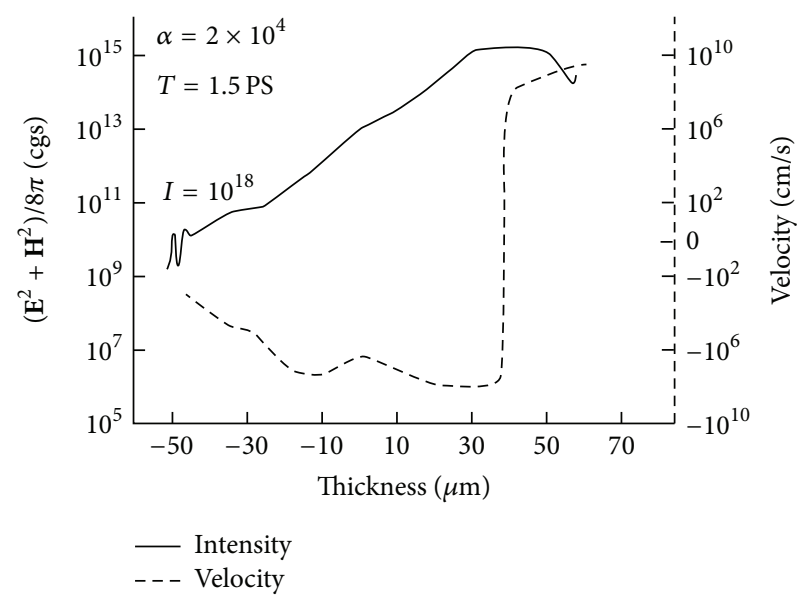

FIgURE 1: Generation of blocks of deuterium plasma moving against the neodymium glass laser light (positive velocities $v$ to the right) and moving into the plasma interior (negative velocities) at irradiation by a neodymium glass laser of $10^{18} \mathrm{~W} / \mathrm{cm}^{2}$ intensity onto an initially $100 \mathrm{eV}$ hot and $100 \mu \mathrm{m}$ thick bi-Rayleigh profile with minimum internal reflection. The electromagnetic energy density $\left(\mathbf{E}^{2}+\mathbf{H}^{2}\right) /(8 \pi)$ is shown at the same time of 1.5 PS after start of the constant irradiation.

will be presented in section 5. Also, Application to a radical new laser driven fusion of solid density fuel without nuclear radiation will be presented in section 6 . Conclusions will be presented in section 7 .

\section{Fusion Energy by Lasers}

It was underlined at the time of the discovery of the laser that irradiation ignition for fusion reactions may be considered using laser radiation. This is based on the fact that Planck radiation of $1 \mathrm{keV}$ temperature has an emission of $10^{17} \mathrm{~W} / \mathrm{cm}^{2}$, an intensity which is well available from lasers. In the following, we describe how some developments to these aims went along three different lines which finally merged in schemes with mutual support.

2.1. Volume Ignition. These studies started with the laser fusion computations using the adiabatic self-similarity model as it was used initially in the computations by Basov and Krokhin, Dawson, Engelhard, and Hora. If an energy $E_{o}$ is uniformly deposited into a volume $V_{o}$ (with radius $R_{o}$ ) of DT of maximum density $n_{o}$ related to the solid state density $n_{s}$, with a break-even energy $E_{B E}$, at an initial zero expansion velocity and an optimum temperature of $17 \mathrm{keV}$, the subsequent adiabatic expansion results in a fusion gain $G$ (DT fusion energy per $E_{o}$, with a break-even energy $E_{B E}$ of $6 \mathrm{MJ})$ :

$$
\begin{aligned}
G & =\left(\frac{E_{o}}{E_{B E}}\right)^{1 / 3}\left(\frac{n_{o}}{n_{s}}\right)^{2 / 3} \\
& =\text { const } n_{o} R_{o} .
\end{aligned}
$$


Formula (1) is based on computations of 1964, published by Hora and Pfirsch in 1970, and the algebraically identical formula (2) by Kidder in 1974. When in these computations the alpha self-heat, the X-ray reabsorption, and the fuel depletion were included, the volume ignition was discovered resulting in very high gains up to 2000 and low ignition temperatures down to $\mathrm{keV}$ and less at compressions to higher than 1000 times the solid state density. This volume ignition was later confirmed by Kirkpatrick and John Wheeler, by Lackner, by Colgate et al., and by others. The most detailed computations by Xiantu $\mathrm{He}$ et al. and Martinez-Val et al. included reheat by neutrons and followed the thermal nonequilibrium between electrons, ions, and the Planckradiation during the very short times of the ignition process such that the very high fusion gains were confirmed. Initial experiments clearly showed that at laser interaction with irradiated solid spheres most of the laser energy uniformly went into a spherical core fully following the self-similarity expansion (a part of the laser energy went into a nonlinear (ponderomotive) force driven fast plasma ablation). It is remarkable that the later measured highest DT neutron generation at direct laser drive exactly followed the selfsimilarity model. Any shock generation had to be avoided (stagnation free Yamanaka compression) in contrast to the shock waves at the fast pusher with much lower gains. The highest gain fusion reactions followed volume burn on the way to volume ignition where the physics is "robust" based on the ideal adiabatic dynamics as it was underlined by Lackner and Colgate et al.

2.2. Radiation Ignition and Spark Ignition. The motivation for this line came from two arguments. First, it was the enthusiasm that the laser irradiation may arrive at similar conditions of radiation ignition in solid or moderately compressed DT fuel as envisaged from the beginning by John Nuckolls based on the radiation ignition or propagating thermonuclear burn in uncontrolled reactions. The second argument was that the gains of (1) were terribly low before the very high gains for volume ignition were discovered. The ingenious approach to solve both questions was the introduction of the spark ignition scheme not long after 1960. The question of directly igniting solid DT by a laser pulse was answered negatively when computations indicated that the fusion flame needs to have an energy flux density of

$$
F>F^{*}=10^{8} \mathrm{~J} / \mathrm{cm}^{2},
$$

or an irradiation corresponding to an ion current density

$$
j>j^{*}=10^{10} \mathrm{Amp} / \mathrm{cm}^{2} .
$$

The condition for beam fusion (4) was by more than five orders of magnitude out of possibilities for ion beams. For achieving condition (3), Nuckolls proposed the spark or central core ignition where the laser radiation (or after conversion into X-rays as "indirect drive") compressed the DT in a very sophisticated way. Isobaric compression should result in a very hot but low density central core whose fusion reaction was then to ignite a very high gain fusion detonation wave into the surrounding low temperature high density mantle. The reaction in the core was shown to be an ideal volume ignition and the energy flux density for driving the fusion detonation wave was in the range of $F=7 \times 10^{8} \mathrm{~J} / \mathrm{cm}^{2}$, fulfilling condition (3). The detailed computation based on otherwise known realistic conditions arrived at generation of $1000 \mathrm{MJ}$ fusion energy produced by a laser pulse of 10 MJ. The problem remains as how to achieve such exclusive temperature and density profiles and how to achieve the necessary orbital symmetry. The study of this spark ignition by indirect drive laser fusion is the aim of the big laser NIF at the Lawrence Livermore National Laboratory. It should be mentioned that parallel to this spark ignition scheme, a pure volume ignition scheme (Section 2.1) leads up to 200 times higher fusion energy per input laser energy by using few MJ ns laser pulses with volume compression to a few thousand times the solid state density and ignition temperatures of $1 \mathrm{keV}$ or even less. This may be considered at least as a very conservative solution for laser driven IFE with the technical task to reduce the size and costs of lasers by many orders of magnitudes. This solution may be possible by physics similar to the reduction of the size of transistors.

2.3. Fast Ignition. When spherical laser irradiation did compress polyethylene-like polymers to 2000 times the solid state density, only a temperature of about $300 \mathrm{eV}$ was measured. In order to reach fusion conditions it was considered to deposit an additional laser pulse of PW power and PS duration at the centre of the compressed plasma. These pulses were just becoming available by chirped pulse amplification (CPA) or the Schäfer technique. However, when performing the first experiments with these pulses, all kinds of relativistic effects were measured: generation of $100 \mathrm{MeV}$ electrons, $\mathrm{GeV}$ ions, $20 \mathrm{MeV}$ gammas, exotic nuclear reactions, pair production, and so forth. This was against the initial aim to deposit the pulse energy at the centre of the 1000 times solid precompressed plasma for spark ignition. Modifications of the fast igniter line were developed of which we mention the laser generation of $5 \mathrm{MeV}$ intense proton beams for depositing energy into the centre of precompressed DT fuel for spark ignition. Another modification by Nuckolls and Wood opens the possibility of the initial aim to ignite nearly uncompressed solid DT fuel. Based on the before mentioned PW-PS laser pulses in 1000 times precompressed plasma, Nuckolls et al. expected that $5 \mathrm{MeV}$ electron beams can be generated with such an intensity that the before mentioned $100 \mathrm{MJ}$ fusion energy may be produced by a $10 \mathrm{~kJ}$ laser pulses. The requirement to produce these fusion gains above 10,000 in a fully controlled way is then fulfilled by using "a large mass of low density DT compressed fuel." The advantage to use the precompression to only 10 times the solid state DT fuel is explained and also how even lower precompression is of an advantage. The use of uncompressed solid state fuel was elaborated in the same sense by using ion beams instead of electrons based on a new scheme where a laser driven plasma block (or piston) ignites the fusion flame. This scheme is described in the next section. 


\section{Laser Driven Ion Beams for Controlled Ignition of Solid DT}

With TW-PS laser pulses a very anomalous new phenomenon was measured, in contrast to the broad stream of observations. This new phenomenon is based on the few interaction measurements which avoid relativistic self-focusing in contrast to the usual experiments where self-focusing results in all kinds of relativistic effects; see above. The essential condition is to use laser pulses with extreme suppression of prepulses, needing a contrast ratio of $10^{8}$ or better. The measured and theoretically understood and numerically reproduced nonlinear force driven plasma blocks with space charge neutralized ion current densities surprisingly reached $10^{11} \mathrm{Amps} / \mathrm{cm}^{2}$ fulfilling condition (4) for igniting nearly uncompressed solid DT similar to the Nuckolls-Wood scheme with electron beams.

3.1. Unique Interaction without Relativistic Self-Focusing. We summarize the essential aspects of these new phenomena. The key question is whether there are conditions under which the interaction of a focused laser beam at the surface of a solid target in vacuum follows the conditions of a plane wave interaction described in one dimension or whether the laser beam, as in most of the usual cases, produces a pregenerated plasma in front of the target, performing relativistic self-focusing and undergoing a shrinking of the laser beam to diameters of a wavelength with subsequent enormous intensities resulting in the relativistic effects. The plane wave interaction was studied by hydrodynamic computations including nearly all realistic and general plasma properties of which one of the numerous cases is shown in Figure 1. Within about a PS interaction of a $10^{18} \mathrm{~W} / \mathrm{cm}^{2}$ neodymium glass laser pulse on a deuterium target with an initially very low reflection (bi-Rayleigh) density profile, the nonlinear (ponderomotive) force is generating blocks of plasma of about 15 vacuum wave lengths thickness moving with velocities exceeding $10^{9} \mathrm{~cm} / \mathrm{s}$ against the laser light and a similar block moving into the target interior.

It was many years later only that these plane geometry conditions were available in experiments, showing such velocities gained by irradiation of similar excimer laser intensities at less than a PS duration, as measured by Sauerbrey. The resulting accelerations of $10^{20} \mathrm{~cm} / \mathrm{s}^{2}$ were in full agreement with the expectation from the nonlinear force interaction. Another key experiment was that by Zhang et al. where $100 \mathrm{fs}$ TW laser pulses of about 30-wave lengths diameter hit a target and the X-ray emission was measured. The laser pulses produced very much lower X-rays than usually known from other experiments with the same intensities. The uniqueness of this experiment was recognized and checked by the procedure that additionally a lower intensity similar pulse was irradiated on the target at varying times from 10 to $100 \mathrm{PS}$ before the main pulse. At short time preirradiation, no change of the low X-ray emission was seen, but as soon as the prepulse time reached 70 PS and more, suddenly the very high X-ray emission appeared as known from all the usual main stream experiments. The later given explanation was

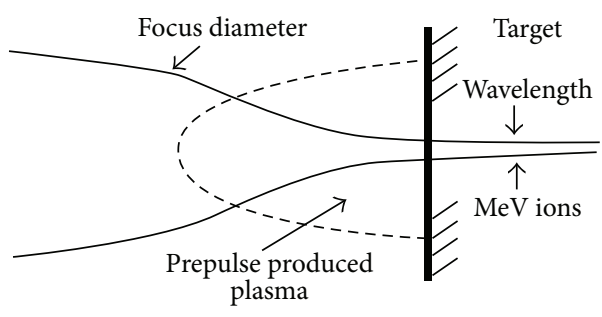

(a)

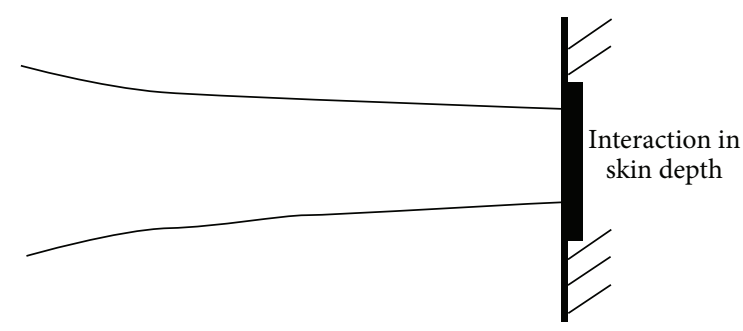

(b)

FIGURE 2: Laser interaction with a target, (a) if the laser pulse produced a plasma plume in front of the target by a prepulse causing shrinking of the beam to less than one wave length diameter due to relativistic self-focusing and (b) avoiding the prepulse for plane geometry interaction within the skin layer.

evident: it was thanks to the clean laser pulse technique where the contrast ratio (ratio of suppression of any prepulse) for the main pulse was $10^{8}$. When the 70 PS prepulse was incident, a plasma plum was generated of a depth about two times the focus diameter. This was sufficient for the main pulse to shrink to about one wave length diameter by relativistic selffocusing so that the very high X-ray intensities resulted as in the usual cases, Figure 2. The skin layer acceleration (SLA) of the plasma block (Figure 2(b)) was just what Sauerbrey observed earlier confirming in retrospect that his $350 \mathrm{fs}$ TW laser pulses were sufficiently clean. The pulses were produced by the Schäfer method amplifying the 350 fs dye laser pulses through an activated $\mathrm{KrF}$ laser medium not needing gratings or pulse compression as with Mourou's chirped pulse amplification (CPA). Sauerbrey measured (by Doppler effect) an acceleration $A$ in a carbon plasma front moving against the laser being produced by a 350 fs TW KrF laser pulse at $3.5 \times 10^{17} \mathrm{~W} / \mathrm{cm}^{2}$ of

$$
A_{\exp }=10^{20} \mathrm{~cm} / \mathrm{s}^{2}
$$

This corresponds to an electric field $\mathbf{E}^{2}=2.9 \times 10^{15} \mathrm{erg} / \mathrm{cm}^{3}$ and a densiy $n_{i} m_{i}$ of the accelerated plasma layer of $5.4 \times$ $10^{-3} \mathrm{~g} / \mathrm{cm}^{3}$ at the critical density $n_{i}=1.6 \times 10^{21} \mathrm{~cm}^{-3}$ for $\mathrm{C}^{+6}$ ions. The nonlinear force for the simplified plane geometry is

$$
\begin{aligned}
f_{\mathrm{NL}} & =-\frac{(\partial / \partial x)\left(\mathbf{E}^{2}+\mathbf{H}^{2}\right)}{8 \pi}=n_{i} m_{i} A \\
& =-\left(\frac{1}{16 \pi}\right)\left(\frac{\omega_{p}}{\omega}\right)^{2}\left(\frac{d}{d x}\right) \mathbf{E}^{2} .
\end{aligned}
$$


Assuming for simplification $d x=\Delta x=10 \mu \mathrm{m}$ and a swelling $S=2$ (the experiments of Badziak et al. for PS pulses resulted in $S=3.5$ ) we find the theoretical value:

$$
A_{\mathrm{NL}}=1.06 \times 10^{20} \mathrm{~cm} / \mathrm{s}^{2} .
$$

Both experiments were a clear confirmation of the plane wave plasma interaction, in agreement with the plane wave interaction theory and computation, Figure 1, and under exclusion of self-focusing. The anomaly was fully realized by the experiments by Badziak et al. from ion emission with high contrast ratio PS-TW laser pulses. This mutually confirmed the high contrast ratio of the excimer laser pulses as well as the very clean techniques of the other experiments. Irradiation of TW-PS neodymium glass laser pulses on copper targets should have led to $\mathrm{Cu}^{+13}$ ions of $22 \mathrm{MeV}$ energy due to relativistic self-focusing, but only $0.5 \mathrm{MeV}$ ions were detected. Furthermore, it was strange that the number of the fast ions did not change at all when the laser intensity was varied by a factor 30 . The energy of the fast ions was fully linear with the laser intensity as expected from a nonlinear force acceleration. The measured X-ray emission leading to a quiver energy swelled dielectrically by a factor three fully satisfactorily agreed with the measured ion energies from a plane wave interaction which happened within the skin depth. The volume of the skin layer was not intensity dependent. This confirmed the observation of the intensity independence of the number of fast ions. These experiments were repeated with gold targets confirming all the details of the skin layer mechanism including the generation of the plasma block, Figure 3, moving into the target as experimentally confirmed from thin irradiated films and confirming an ion current density in the blocks above:

$$
j>10^{10} \mathrm{~A} / \mathrm{cm}^{2} \text {. }
$$

The measurements confirmed also that the PS-TW interaction resulted only in one narrow ion beam accelerated against the laser light as expected from the nonlinear force acceleration while the use of longer laser pulses as usually resulted in several groups of ions moving into a wide angle against the laser light. Now we report some details including recent numerical results for deuterium plasmas. The computations are based on the fact that before the PS main pulse, a less than 50 PS earlier acting laser prepulse produces a preplasma layer of thickness $L_{\text {pre }}$ at least several times smaller than the laser focal spot diameter $d_{f}$. The main laser pulse interacts most intensively with the plasma in the skin layer near the surface of the critical electron density $n_{e c}=m_{e} \omega^{2} / 4 \pi e^{2}(\omega$ is the laser frequency) and the geometry of the interaction is almost planar $\left(L_{\text {pre }} \ll d_{f}\right)$. The high plasma density gradient in the interaction region produces nonlinear ponderomotive forces acting, at the laser beam incidence perpendicular to the target surface, nearly parallel to the target normal. The force density $f_{\mathrm{NL}}$ can be expressed as the one-dimensional negative gradient of the electromagnetic energy density of the laser field given by its (dielectric modified) electric and magnetic vectors $\mathbf{E}$ and $\mathbf{H}$ :

$$
f_{\mathrm{NL}}=-\frac{(\partial / \partial x)\left(\mathbf{E}^{2}+\mathbf{H}^{2}\right)}{8 \pi} .
$$

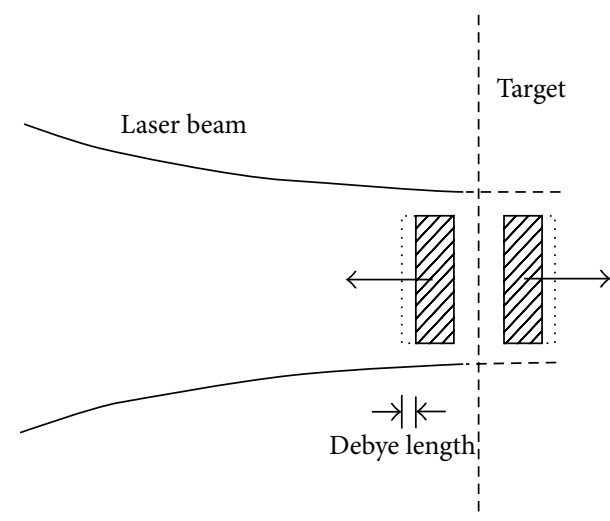

FIGURE 3: Scheme of skin depth laser interaction where the nonlinear force accelerates a plasma block against the laser light and another block towards the target interior. In front of the blocks are electron clouds of the thickness of an effective Debye length of less than $500 \mathrm{~nm}$.

The gradients of the energy density near the critical surface result in two opposite nonlinear forces which break the plasma and drive two thin $(\sim$ few $\lambda)$ plasma blocks towards the vacuum and towards the plasma interior, Figure 3 , respectively ( $\lambda$ is the laser wavelength). The density of the plasma blocks is high (the ion density $n_{i} \approx n_{e c} / z$, where $z$ is the ion charge state) but the electron temperature is fairly moderate at subrelativistic laser intensities. Thus, the Debye length, $\lambda_{D}$, is small $\left(\lambda_{D} \ll \lambda\right)$ inside the block and ions, being closely attached to electrons, move together with the electron cloud driven directly by the ponderomotive force. Since $n_{i} \sim 10^{21}-10^{22} \mathrm{~cm}^{-3}$ at $\lambda \sim 0.3-1 \mu \mathrm{m}$, even at moderate ion velocities $v_{i} \sim 10^{7}-10^{8} \mathrm{~cm} / \mathrm{s}$, the ion current densities can be very high $\left(\sim 10^{9}-10^{10} \mathrm{~A} / \mathrm{cm}^{2}\right.$ or higher). The computations used the genuine two-fluid model and were performed for a $20-\mu \mathrm{m}$ hydrogen, inhomogeneous plasma layer of initial density increasing in the direction of the laser beam propagation. Both the linear plasma density profile and the profile described by the function $n_{e}(x)=2 \times n_{e c}\{1+$ $\left.\exp \left[\left(x-x_{c}\right) / 05 \cdot L_{n}\right]\right\}^{-1}$ were considered as initial values. For both profiles the boundary plasma densities were chosen in such a way that the critical plasma density $n_{e c}$ was placed near the middle of the layer. The initial inhomogeneity of the plasma was characterised by the (relative) plasma density gradient scale length, $L_{n} / \lambda$, where $L_{n}=n_{e c}\left(\partial n_{e} / \partial x\right)^{-1}$. The initial temperatures of both electrons and ions were assumed to be of $30 \mathrm{eV}$. Most of the calculations were carried out for laser pulses of a Gaussian shape and for a laser wave length $\lambda=1.05 \mu \mathrm{m}$. Our numerical studies were focused on the influence of the initial plasma inhomogeneity and the laser pulse parameters (intensity, duration) on the plasma characteristics and, particularly, on the current densities and velocities of ion fluxes produced by the laser-plasma interaction.

The following results are for the nonlinear initial plasma density profile (the results for the linear profile were qualitatively similar). Figure 4 represents spatial distributions of 


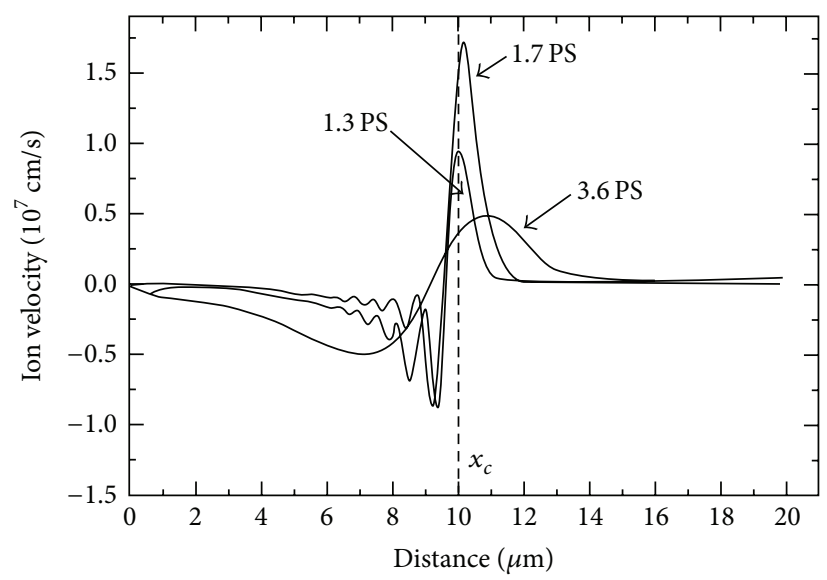

(a)

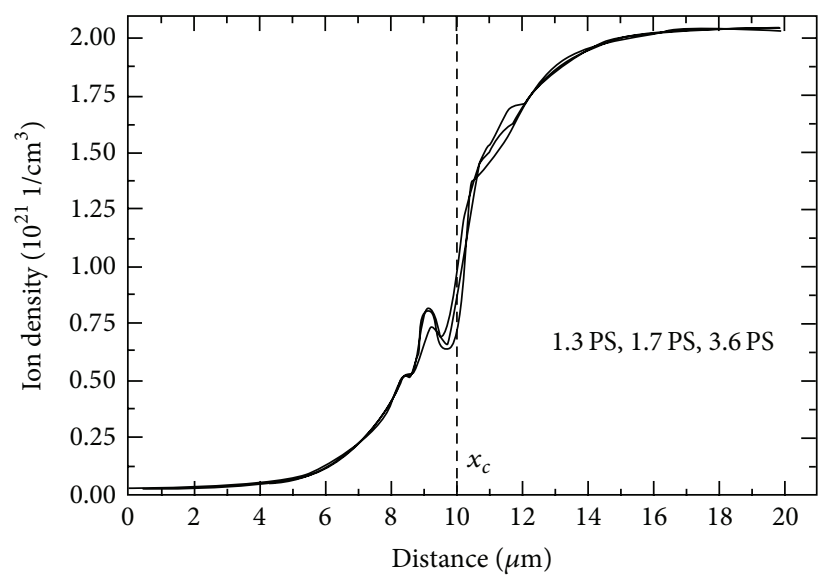

(c)

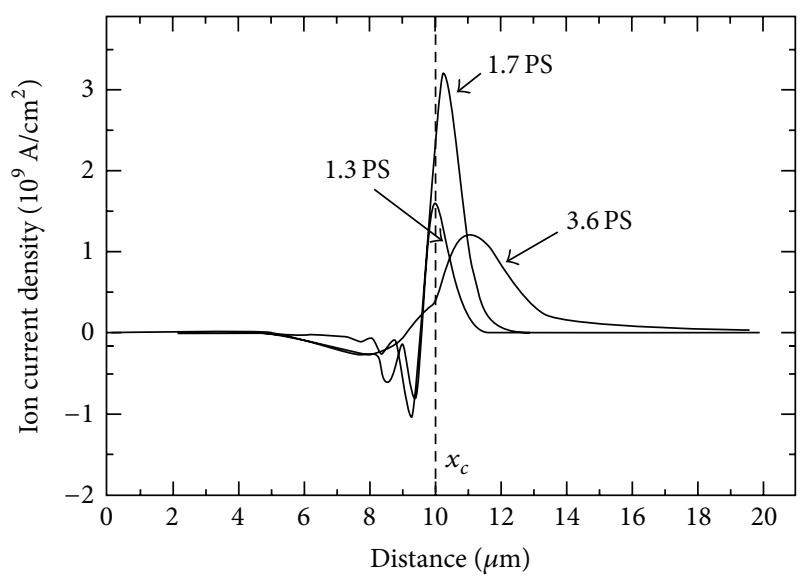

(b)

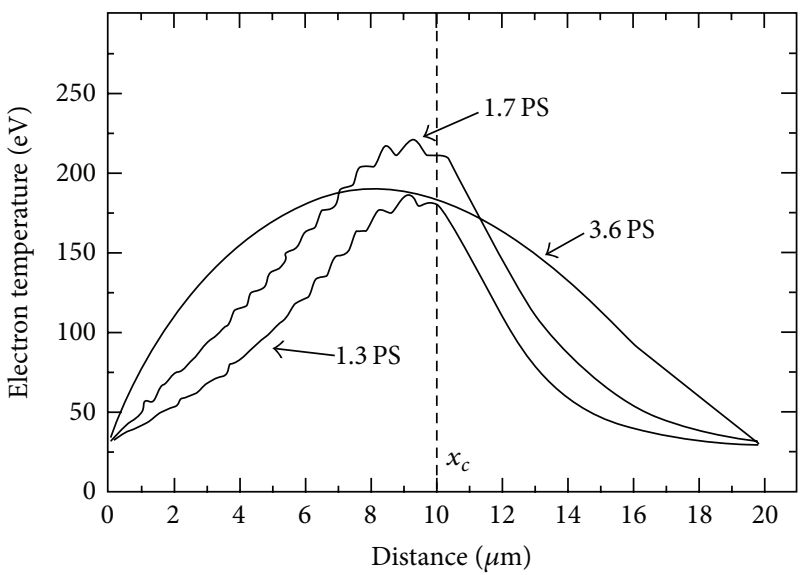

(d)

Figure 4: Spatial distributions of the ion velocity (a), the ion current density (b), the ion density (c), and the electron temperature (d) of plasma for various times measured from the beginning of the laser-plasma interaction. $\tau_{L}=1 \mathrm{PS}, I_{L}=10^{16} \mathrm{~W} / \mathrm{cm}^{2}, L_{n} / \lambda=2.5$.

the ion velocity, the ion current density, the ion density, and the electron temperature of plasma for the three-time intervals after the beginning of the laser-plasma interaction. We see the generation of the under dense plasma block moving against the laser (negative velocities and current densities) and the over dense plasma block behind the critical surface moving in forward laser direction (positive velocities and current densities) in accordance with the simple physical picture. The plasma density profile is significantly disturbed near the critical surface and the electron temperature is the highest there. The effect of the plasma density gradient scale length on the maximum ion current densities and the maximum ion velocities of backward- and forward-emitted ion fluxes can be seen in Figure 5. For both fluxes there exist optimum values of $L_{n} / \lambda$ but they are located in essentially different regions of $L_{n} / \lambda$ : the highest current densities and velocities for the backward flux are attained at the small density gradients and for the forward flux at the high density gradients.
The influence of laser pulse duration, $\tau_{L}$, on characteristics of ion fluxes is illustrated in Figure 6. For the forwardemitted ions, the maximum ion velocity and the maximum ion current density continuously increase with an elongation of the laser pulse. Contrary to that, in the case of backwardemitted ions, distinct maxima of these quantities occurs at $\tau_{L}$ sub-PS ranges. One of the characteristic features of the dependencies presented in Figures 5 and 6 are the essential differences in the run of these dependencies for forwardand backward-emitted ion fluxes. These differences can be understood better when we consider the dielectric swelling factor $S=1 /|n|$ which is a measure of increase in the electromagnetic energy density of the laser field in plasma in relation to its vacuum value ( $n$ is the plasma refractive index). The necessary condition for the acceleration of plasma (ions) by the ponderomotive force in the backward direction is $S>1$ and, usually, the higher $S$, the higher the backward-directed ponderomotive force. The swelling factor depends not only on the plasma density gradient but also on parameters of 


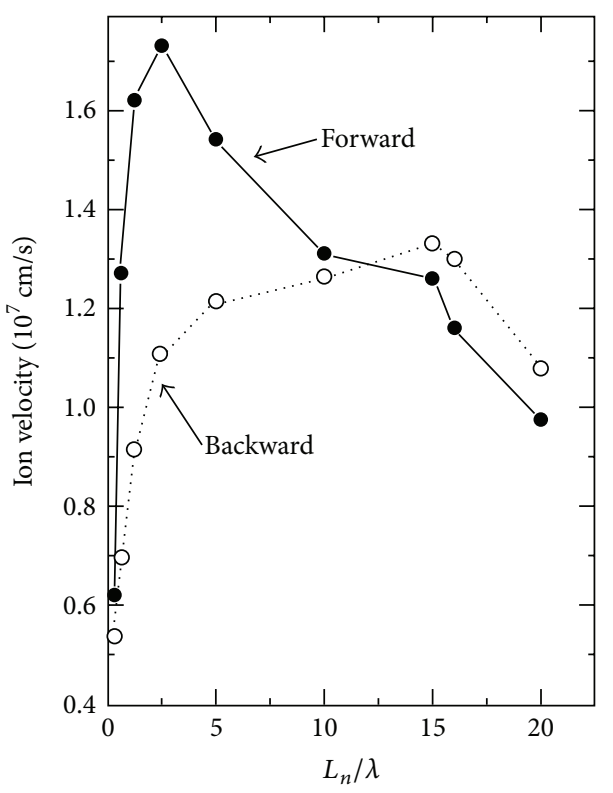

(a)

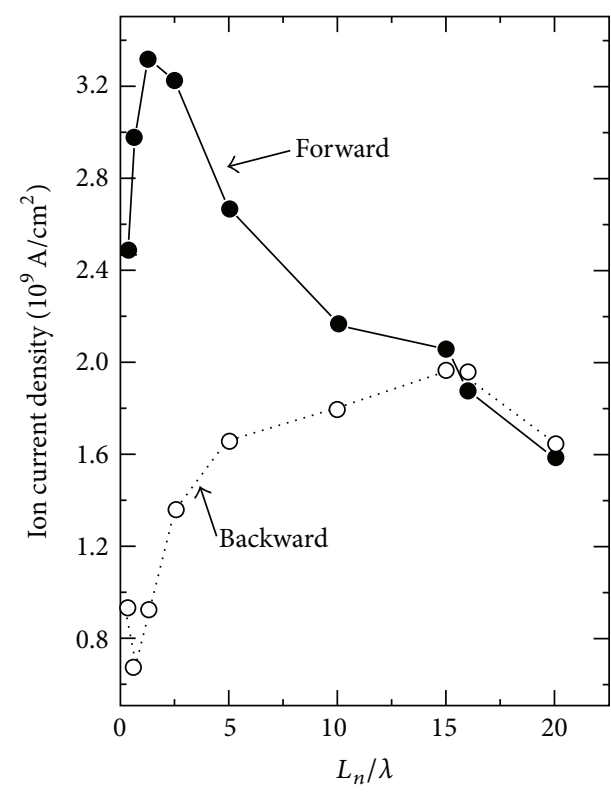

(b)

Figure 5: The dependencies of the maximum ion velocities (a) and the maximum ion current densities (b) on the plasma density gradient scale length. $\tau_{L}=1 \mathrm{PS}, I_{L}=10^{16} \mathrm{~W} / \mathrm{cm}^{2}$.

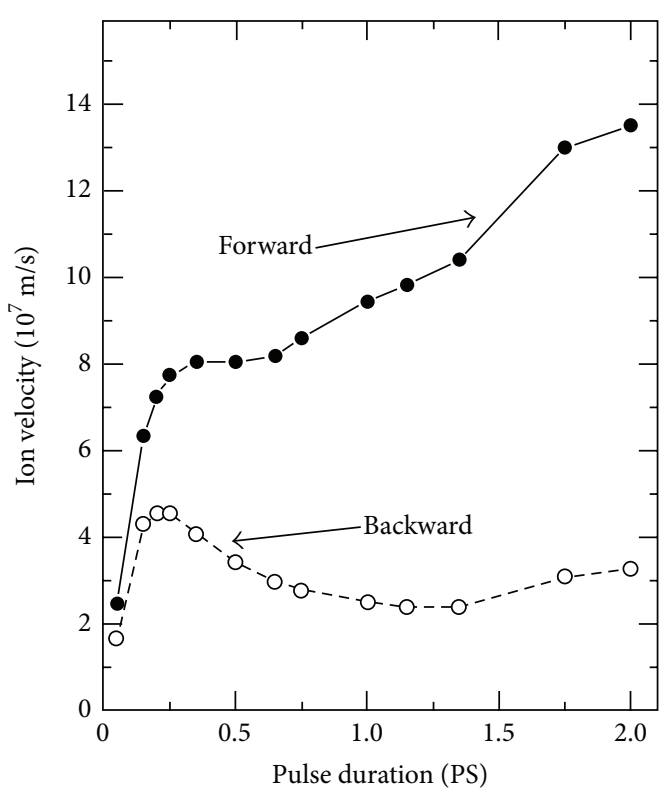

(a)

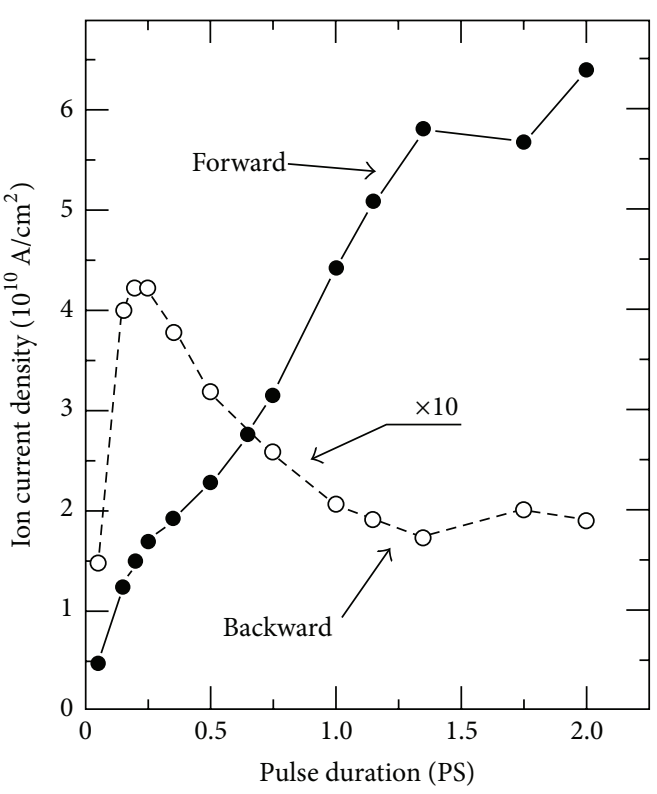

(b)

Figure 6: The maximum ion velocities (a) and the maximum ion current densities (b) as a function of the laser pulse duration. $I_{L}=$ $10^{17} \mathrm{~W} / \mathrm{cm}^{2}, L_{n} / \lambda=1$.

a laser pulse and particularly on its intensity and the pulse duration. As it results from our numerical simulations, the swelling factor (its maximal value) decreases when the plasma density gradient $\lambda / L_{n}$, the intensity, or the pulse duration increase (within the ranges of these parameters shown in the figures). For instance, at $L_{n} / \lambda=1, I_{L}=10^{17} \mathrm{~W} / \mathrm{cm}^{2}$, the swelling factor decreases from $S \approx 2$ for $\tau_{L}=0.2$ PS down to $S \approx 1.3$ for $\tau_{L}=2$. Thus, at high values of the above three parameters $\left(\lambda / L_{n}, I_{L}, \tau_{L}\right)$, the forward-directed force from the usual light pressure predominates the backward-directed 
one and, as a result, the velocities and current densities of forward-emitted ions are considerably higher than the ones for backward-emitted ions. The decreasing swelling factor is also the main reason for the faster increase in $v_{f}$ and $j_{f}$ (than $v_{b}$ and $j_{b}$, resp.) when the laser intensity increases. The block generation was reproduced also by an essentially different genuine two-fluid computation, Figure 7. In conclusion, the numerical simulations based on the two-fluid hydrodynamic plasma model confirmed both qualitatively and quantitatively the idea of production of ultrahigh- currentdensity ion beams with the use of ponderomotive forces induced at the skin layer interaction of a short laser pulse of subrelativistic intensity with a thin inhomogeneous plasma layer. The results of the computations were found to be consistent with the simplified theory and measurements.

3.2. Application for Controlled Ignition. Since the ion current densities, (6), in the laser driven plasma blocks from the skin layer or corona area of the irradiated plasma fulfilled the condition (4) for ignition of solid DT, we evaluated what has been achieved experimentally with respect to the energy flux density $F$, (3). The measurements included values of $F$ up to $10^{6} \mathrm{~J} / \mathrm{cm}^{2}$. If the ignition of DT has to use ion energies of $80 \mathrm{keV}$ for the optimum resonance conditions this gives an upper limit for the laser intensity while simultaneously a minimum of $F$ is necessary. The values of $F^{*}=10^{8} \mathrm{~J} / \mathrm{cm}^{2}$ may be not the final answer. It has been discussed before that the detailed evaluation of the interpenetration of the energetic blocks with the cold solid DT may result in 20 times lower values

(i) if the increased collision frequency based on the quantum correction is included,

(ii) if the modification of the stopping power by collective effects is included, and

(iii) if the double layer caused reduction of the thermal conduction is taken into account.

But even with the pessimistic aim to work with $F^{*}$ values above $10^{8} \mathrm{~J} / \mathrm{cm}^{2}$, this can be reached with short wave length lasers, for example, the double frequency of $\mathrm{KrF}$.

The remaining problem is only whether the interaction mechanisms of the plasma block of sufficient thickness is comparable to the interaction process as in the case of the electron beam in the Nuckolls-Wood scheme; otherwise, there is the similarity to the spark ignition at the interaction of the hot core with the surrounding plasma at spark ignition. For achieving the necessary thickness of the block, one has to produce it by irradiating a solid thin DT layer of spherical shape where the compressing part is then ballisticly moving into a focus for the interaction with the main solid DT fuel. During this motion, there is an increase of the density of the fast shell. Sine a minor heating is unavoidable apart from the nonlinear force driven directed motion, the shell is a little expanding thermally. But in view of the spherical shrinking of the shell, the final interaction occurs with the same high ion current density needed for conditions (4) however in a block of increased thickness needed for the ignition. This ballistic increase was mentioned before for the proton

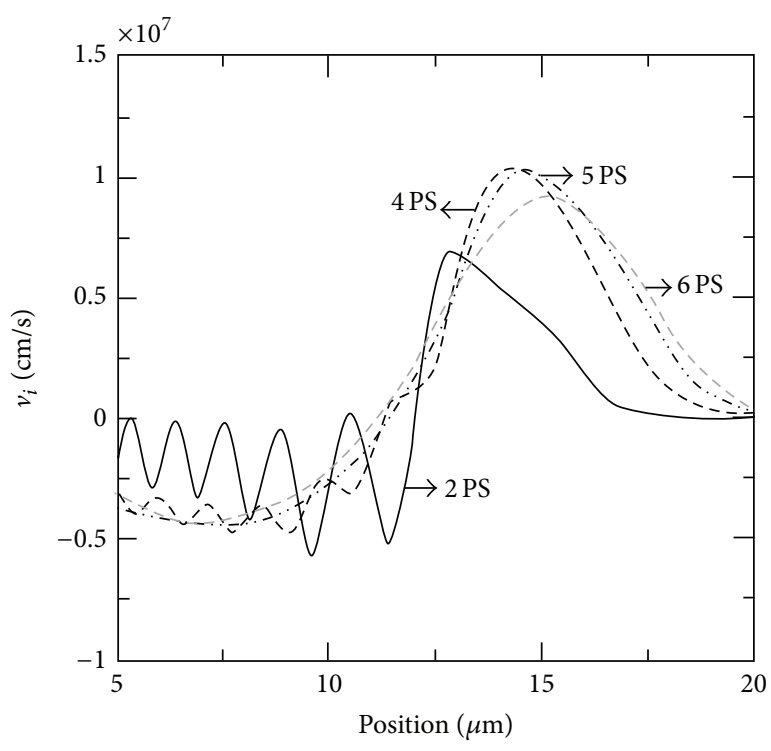

FIGURE 7: Ion velocity profiles at times 2, 4, 5, and 6 PS after irradiation of a 4 PS neodymium glass laser pulse on a deuterium plasma of initial density of a linearly increasing ramp (abscissa in micrometer of the depth, with critical density at 13) of $30 \mathrm{eV}$ temperature, confirming the generation of an ablating plasma block (negative velocity) and a compressing plasma block (positive velocity).

ignition generated by PW-PS laser pulses in precompressed DT fuel. It was shown that the ion current densities in plane geometry can be 1000 times higher than known before from measurements without the plasma block acceleration. With this result alone the scheme of proton fast ignition should reach the conditions of high gain fusion reactions. If then the ballistic layer acceleration is used to be focused into the main fuel, the conditions for ignition again will be improved. In this case indeed the ion energy for the ignition is not in the range of $80 \mathrm{keV}$ as in the before mentioned ignition of solid DT fuel, but the ion blocks have to be relativistic with ion energies in the $5 \mathrm{MeV}$ range. The details of this block acceleration have been evaluated numerically. In all our computations we used the genuine two fluid hydrodynamics including collisions. It is remarkable that the very different collision-less treatment with PIC methods resulted in very similar results at comparable conditions. The plasma block interaction for highly relativistic laser intensities was studied also in view of fusion ignition and further applications. Application of plasma block acceleration for improving the proton-fast-ignition fusion scheme with 1000 times higher ion current densities from the block acceleration was shown in the Figure 8.

\section{Sauerbrey's Experimental Discovery of Ultrahigh Laser Acceleration of Plasma by Nonlinear Forces}

Sauerbrey's [21] measurement of ultrahigh acceleration of plasma blocks by lasers turned out to be basically a nonthermal interaction process for which clarification was needed 


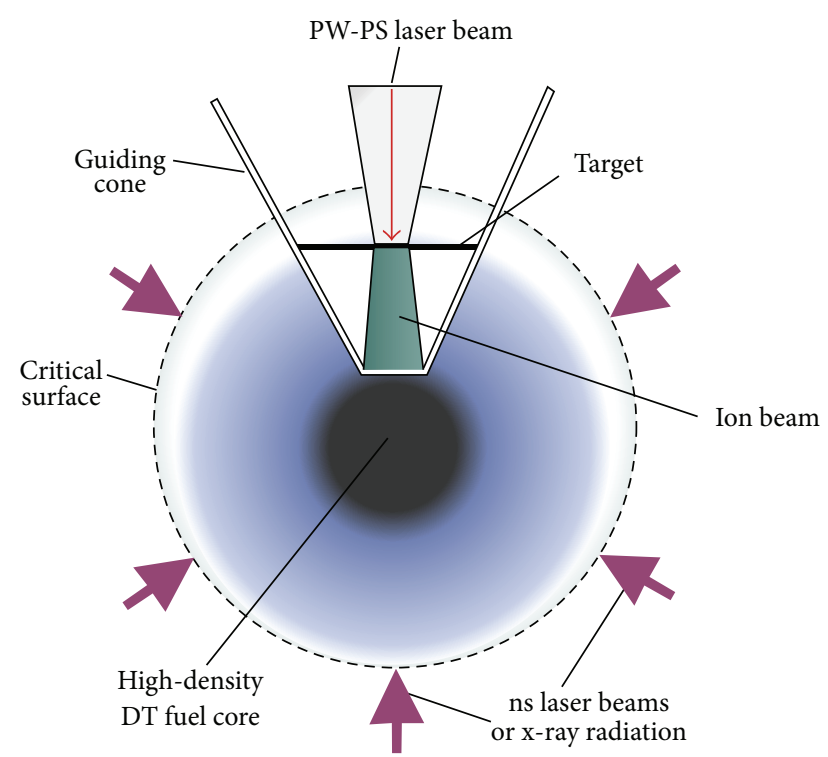

FIGURE 8: Application of plasma block acceleration for improving the proton-fast-ignition fusion scheme with 1000 times higher ion current densities from the block acceleration.

and developed in due course, as the problems were of a rather complicated nature. First we give the final short-cut result before going into details. The final result in hydrodynamic plasma theory is based on the force density $f$ in a plasma to be determined by the gas-dynamical pressure $p=3 n_{p} k T / 2$ where $n_{p}$ is particle density, $k$ is Boltzmann's constant, and $T$ is the temperature and by the present electric and magnetic fields $\mathbf{E}$ and $\mathbf{H}$ :

$$
\mathbf{f}=-\nabla p+\mathbf{f}_{\mathrm{NL}}
$$

For fields of a laser of frequency $\omega$ defining a complex optical constant $\mathbf{n}$ in the plasma, the nonlinear force with the unity tensor 1 is

$$
\begin{aligned}
\mathbf{f}_{\mathrm{NL}}= & -\left(\nabla \cdot \left[\mathbf{E E}+\mathbf{H H}-0.5\left(\mathbf{E}^{2}+\mathbf{H}^{2}\right) \mathbf{1}\right.\right. \\
& \left.\left.+\left(1+\frac{(\partial / \partial t)}{\omega}\right)\left(\mathbf{n}^{2}-1\right) \mathbf{E E}\right]\right)(4 \pi)^{-1} \\
& -\left(\frac{\partial}{\partial t}\right) \mathbf{E} \times \frac{\mathbf{H}}{4 \pi c}
\end{aligned}
$$

which is dominant over the gas dynamic force (also called the thermokinetic force $\mathbf{f}_{\mathrm{th}}=-\nabla p$ ) if the quiver energy of the electrons in the laser field is larger than the energy of thermal motion (Hora 1969; 1985; see (8.87) and (8.88) of Hora [23]). For simplified one-dimensional geometry and perpendicular laser irradiation, the force (2) can be reduced to the time averaged value [24]:

$$
\mathbf{f}_{\mathrm{NL}}=-\frac{(\partial / \partial x)\left(\mathbf{E}^{2}+\mathbf{H}^{2}\right)}{8 \pi}=-\frac{\left(\omega_{p} / \omega\right)^{2}(\partial / \partial x)\left(E_{v}^{2} / \mathbf{n}\right)}{16 \pi},
$$

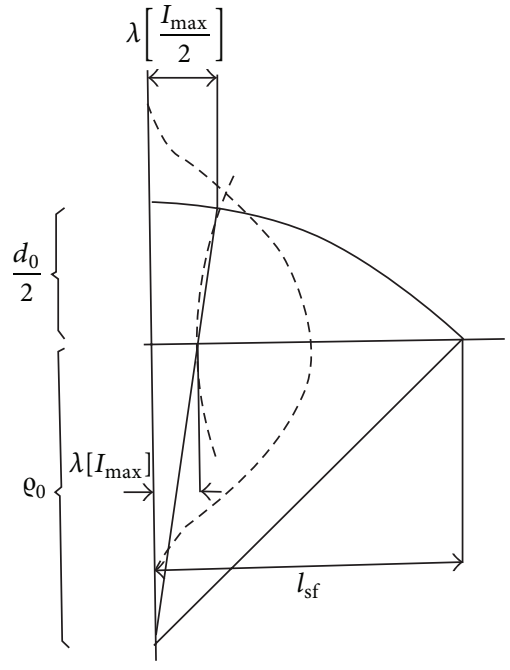

FIGURE 9: Relativistic self-focusing [26]: relativistic change of electron mass results in shorter optical propagation at higher intensity than at lower intensity. Relativistic self-focusing of a laser beam from the left with a dashed Gaussian intensity profile and radius $d_{o} / 2$ moves into plasma right from the vertical line. Due to the relativistic change of the electron mass at the quiver motion, the effective wave length within the plasma is dielectrically shorter at higher intensity then at lower causing a concave bending of the initially plane wave front with shrinking of the beam to less than wave length diameter $[26,31,32]$.

where $E_{v}$ is the amplitude of the electric field of the laser in vacuum. The second expression corresponds to the formulation of the ponderomotive force in electrostatics while the first expression includes identities from the later Maxwellian theory with the magnetic field as shown before [23]. Computations for plane geometry interaction with inclusion of the nonlinear force interaction, with thermal laser absorption by collisions and equipartition processes in the dynamically developing optical plasma properties for interaction of neodymium glass laser irradiation of $10^{18} \mathrm{~W} / \mathrm{cm}^{2}$ intensity on deuterium having an initial doubleRayleigh density profile (see Hora [25], Figures 9 and 10), arrived at a velocity distribution and an electromagnetic energy density as shown in Figure 1 after 1.5 PS interaction time. The laser was irradiating from the right hand side and a plasma block was moving against the laser light and another one into the deeper target. The velocity at this very short time at the closest part to the laser was more than $10^{9} \mathrm{~cm} / \mathrm{s}$. This corresponds to an average acceleration of more than $5 \times 10^{20} \mathrm{~cm} / \mathrm{s}^{2}$.

These results of the computation were initially published in 1978 (see [25]) but it took a long time [21] before an experimental confirmation of these ultrahigh accelerations was measured. The reason was not only the question of how to produce the PS laser pulses up to the range of terawatt (TW) power, but there was the difficulty of relativistic self-focusing [26]. Each laser prepulse produced a plasma plume where any very intense laser beam which was relativistically squeezed to less than wave length diameter, Figure 9, producing very high intensities resulting in emission of highly charged 


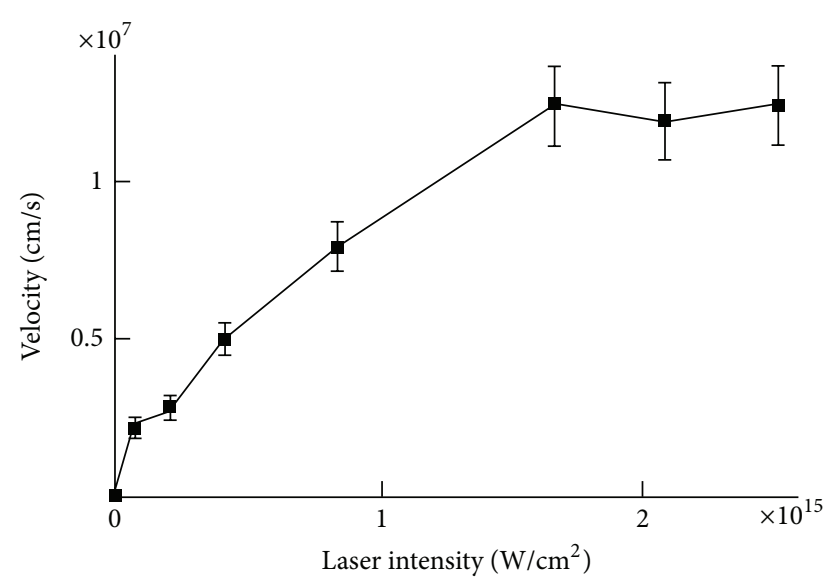

FIGURE 10: Intensity dependence of the velocity of the plasma front from the Doppler shift of the reflected $700 \mathrm{fs} \mathrm{KrF}$ laser pulses from Al target [37].

ions to energies far above $\mathrm{MeV}$ and extremely intense very short wave length $\mathrm{X}$-rays. These extreme mechanisms due to relativistic self-focusing had to be suppressed for verifying the plane geometry of the early computations and also to provide the conditions that the ultrahigh acceleration could be detected directly by Doppler effect. A confirmation of these conditions in the measurements of Sauerbrey [21] and the nonthermal processes was supported by following experiments $[27,28]$, after similar measurements led to the conclusion that relativistic self-focusing was suppressed and shown from further related experiments $[1,2,29]$. This was in drastic contrast to the usual experiments with PS-TW and few petawatt (PW) laser pulses studied by the broad stream of experiments after Cowan et al. [30] observed high acceleration of ions and electrons, pair production, gamma rays with subsequent nuclear transmutations, and so forth. The characteristic for the very rare experiments of Sauerbrey [21], Zhang et al. [27], and Badziak et al. [28], see Hora et al. [2], is the use of the extremely high contrast ratio above $10^{8}$ for the suppression of the relativistic self-focusing for achieving the enormous suppression of laser prepulses before the arrival of the main pulse on the targets. The reason for this was a specific need with the unique KrF laser pulses used by Sauerbrey [21] where subpicosecond laser pulses from dye lasers propagated in an inverted $\mathrm{KrF}$ laser gas for pulses reaching nearly TW power $[19,20]$. The $\mathrm{KrF}$ excimer wave length of $248 \mathrm{~nm}$, in contrast to the longer laser wave lengths of the usually applied lasers, was disturbing the experiments by the amplified spontaneous emission (ASE) radiation where the short wave length was preionizing the target too much. It was therefore highly necessary to reduce the ASE to a sufficient degree by suppression of any prepulse using plasma mirrors and other techniques for this type of lasers [20]. Sauerbrey therefore used lasers with a cutoff of the prepulses by a factor above $10^{8}$ (contrast ratio) and had an interaction of the plane one-dimensional wave front of the interacting laser avoiding any self-focusing. The resulting plane wave front geometry provided then for the very first time the conditions for which the interaction was calculated before
(Figure 1). Sauerbrey measured an acceleration of the plane plasma fronts perpendicular to the target of $10^{20} \mathrm{~cm} / \mathrm{s}^{2}$ against the laser as immediately given from the Doppler shift. This was in full agreement with the nonlinear force acceleration as computed before. Variations with respect to experimental accuracy were of minor nature for comparison in view of the significant fact that these ultrahigh accelerations were 100,000 times higher than the thermal pressure acceleration with the largest NIF (National Ignition facility) laser (Park et al. 2010) using nanosecond laser pulses.

\section{Analysis of Acceleration Measurements with Doppler Effect}

Compared with the Doppler experiments with $\mathrm{KrF}$ lasers, the continuation with solid state lasers indicated a number of complexities which still have to be studied. It was possible [33] that a suppression of relativistic self-focusing for generation of plane plasma blocks as known from the numerous experiments with solid state lasers by Badziak et al. $[34,35]$ was possible by the proof of very low emission of X-rays [36] following the method of Zhang et al. [27]. Doppler experiments were performed with $\mathrm{KrF}$ PS laser pulses [37-39] which are now discussed in view of the later reached clarification between thermal and nonlinear force acceleration processes. Figure 10 shows the measured plasma block velocities from the Doppler effect at varying $\mathrm{KrF}$ laser intensities for $700 \mathrm{fs}$ laser pulses with sufficiently high contrast ratio to suppress the ASE. The laser pulse of $2.6 \times 10^{15} \mathrm{~W} / \mathrm{cm}^{2}$ intensity produced a velocity of $1,25 \times$ $10^{7} \mathrm{~cm} / \mathrm{s}$ of the plasma at irradiation of aluminium [37]. This corresponds to an acceleration of $1.6 \times 10^{19} \mathrm{~cm} / \mathrm{s}^{2}$. The question is then how fast the laser energy can be converted into plasma motion. At this intensity, the quiver energy of electrons

$$
\varepsilon_{\mathrm{osc}}=\frac{e^{2} \mathbf{E}_{v}^{2}}{\left(2 m_{e} \omega^{2}\right)}
$$

using the electron mass $m_{e}$ is $31.2 \mathrm{eV}$ with the electric field amplitude $\mathbf{E}_{v}$ of the laser in vacuum for the KrF laser. This is sufficient to produce plasma electrons in the target within about a laser period by the electrons impacting in the target and with other aluminium ions to be ionized. The generation of an inhomogeneous plasma density in the interaction region can well be assumed to be within this very short time to produce a dielectric swelling $S=3$ which determines the electric field $\mathbf{E}$ by dielectric properties given by the temporary and spatially change during the: interaction dynamics by the absolute value of the refractive index $\mathbf{n}$ :

$$
E^{2}=\frac{E_{v}^{2}}{\mathbf{n}} \quad S=\frac{1}{\mathbf{n}} .
$$

This number $S$ can be larger or smaller. The experience from the evaluation of the experiments of Badziak et al. [28] arrived at a swelling of a value of 3 [29]. The example of Figure 1 of the numerical velocity of deuterium with an assumed initial Rayleigh density profile had a swelling above $S=15$. 
Swelling by $S=3$ produced a quiver energy of the electrons in the considered case for aluminium within fs duration had an energy of $93 \mathrm{eV}$ and may then have produced high ionization $Z$ of the aluminium. The dynamics of the plasma will then be determined by the nonlinear force (3) acting on the electron cloud again within the time of quiver motion to an acceleration whose dynamics will be determined by the inertia of the ion cloud due to electrostatic attraction in the same way as in the example of Figure 1 in agreement with the experiments of Sauerbrey [21]. Taking full ionization $Z=13$, the front of the fastest ions arrives at a velocity of $1.2 \times 10^{7} \mathrm{~cm} / \mathrm{s}$. In view of a possible higher number $S$ or lower number $Z$, it is sufficient at this stage only to see that the $700 \mathrm{fs}$ laser interaction is sufficient to produce the measured acceleration. This is in contrast to any thermal mechanism, where first the quiver energy of the electrons has to be changed by collisions into random motion determining a temperature which then by a rather long equipartition time leads to heat the ions to move with the measured high plasma velocity. Similar acceleration velocities from Doppler experiments by Veres et al. [38] agree with the considered results from aluminium where the intensity dependence in comparison with the experiments of Badziak et al. [28] and the difference between irradiation of polystyrene and of aluminium may be interpreted along the discussed results of Földes at al. [37]. At oblique incidence of lasers on the target, the nonlinear force is directed perpendicular to the plasma surface (see Section 11 of [23]) and the laser field energy density is not drastically modified. The acceleration of the ions is happening directly by the conversion of the laser field energy into the nonlinear force driven electron cloud driving the ion cloud by electrostatic attraction. This is in contrast to thermal absorption and heating which takes a much longer time than the interaction of the PS laser pulses. Computations at plasma surfaces with similar initial Rayleigh density profiles as in Figure 1 were performed, however, using a later developed genuine two-fluid hydrodynamic computation scheme. This code was developed by using the Euler equations of electrons and for ions separately combined by the electric fields given by the Poisson equation. These internal electric fields in the plasmas were eliminated in Schlüter's [40] two-fluid hydrodynamics where the Euler equations were added to arrive at a one-fluid plasma equation of motion (1) used in the computations of Figure 1, and where the difference of the of the Euler equations led to a generalized Ohm's law for the plasmas. This elimination of the electric fields was correct for homogeneous, uniform plasmas similar to metals where the electric conductivity is similar to plasmas and where any electric field is decaying in much shorter times than femtoseconds (fs). For dimensions where the Debye length is much shorter than the considered length dimensions, the electric fields can well be ignored too. If these fields did appear in extraterrestrial plasmas as known by Alfven [41] and his associates, it was mentioned in a book review about this work by Kulsrud [42] that "these fields are intuitively not clear." In contrast to these general views in plasma theory, the genuine two-fluid hydrodynamics showed the strong appearance of these electric fields especially in the highly inhomogeneous plasmas at very intense laser

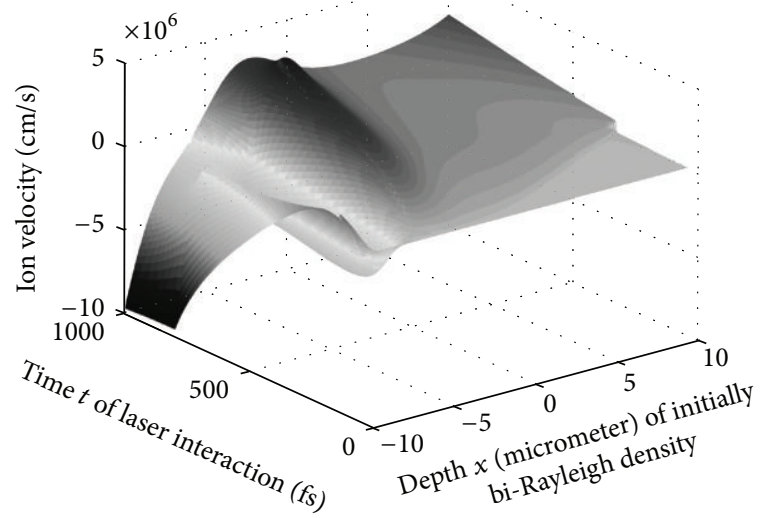

FIGURE 11: Ion velocity within an initially bi-Rayleigh $\alpha=9.59 \times$ $10^{3} \mathrm{~cm}^{-1}$ deuterium plasma with initially zero velocity and with $100 \mathrm{eV}$ initial temperature located between $x=10$ and $+10 \mu \mathrm{m}$ at neodymium glass laser irradiation from the left hand side of $2 \times 10^{16} \mathrm{~W} / \mathrm{cm}^{2}$ intensity during the time between 0 and $750 \mathrm{fs}$ [47].

interaction ([43, 44]; see Sections 8.8, 8.9, and 10.7 of [23]). It was then most interesting to see how these electric fields modify the results of Figure 1. After the genuine two-fluid code was applied to study the experiments of Badziak et al. [35] using genuine two-fluid hydrodynamics $[45,46]$ and other details of the nonlinear force dominating the laserplasma interaction (Cang et al. 2006), a numerical evaluation was achieved for the initially Rayleigh plasma density profiles similar to Figure 1 [47], where high swelling factors, $S$, were again the result. It turned out that the net ion motion was not much changed, Figure 11, compared with Figure 1 due to the fact that there was a dominant nonthermal conversion of laser energy into the macroscopic ion motion by the nonlinear force. The very general code with inclusion of collisions for thermal absorption and equipartition for ion heating were highly delayed and not strongly affecting the plasma dynamics determined by the ions for the very high laser intensity interactions during pulses in the PS range.

\section{Application to a Radical New Laser Driven Fusion of Solid Density Fuel without Nuclear Radiation}

What was important with the ultrahigh acceleration was that extremely high current densities in the highly directed space charge neutral plasma blocks arrived at $10^{11} \mathrm{Amps} / \mathrm{cm}^{2}$ or more. This is again more than a million times higher than accelerators could provide for ion beam fusion and permitted a comeback of the reaction of solid state, uncompressed or modestly compressed, fusion fuel by side-on ignition of a fusion flame. This was absolutely impossible with the first side-on ignition calculations for solid density fusion fuel (Chu 1971), [48] but this has changed now with the $>$ PWPS laser pulses $[18,30]$. It is potentially possible for energy production in power stations to achieve gains of 10,000 
similar to the Nuckolls and Woods [49] scheme using PSlaser produced very high density $5 \mathrm{MeV}$ relativistic electron beams in analogy to the here treated nonlinear force driven plasma blocks based on ions. The theory of Chu (1971) had to be updated [50] by the then not known effects of the thermal inhibition factor and by the collective effect of the stopping power (Gabor 1953). For laser fusion of deuterium tritium (DT), extremely clean PS laser pulses with a contrast ratio above $10^{8}$ may drive the controlled reactions in power stations with pulses in the range of few dozens of PW power (Hora 2009). These are close to technical realization. What was very surprising is that the reaction of hydrogen and the boron isotope 11 (HB11) is less than ten times only more difficult than the DT fusion. This will generate less radioactivity in the entire reaction and in the waste than burning coal, per energy production $[51,52]$. In contrast to the need of extremely high fuel compression in the usual thermally ignited laser-fusion schemes, the side-on ignition is simplifying the process and it can be expected that power production can be at considerably lower cost than present lowest cost sources, as today's cost in Eurocents/kWh: nuclear fission 2, coal 5, wind 8 , and photovoltaics 38 . For the next exploration of the side-on ignition of laser fusion with nonlinear force driven plasma blocks, the initial computations (Hora 2009; Hora et al. 2009) [51] are now generalized to use the genuine two-fluid model $[43,44]$ in order to study details of shock generation and very high electric field dynamics in the extremely inhomogeneous plasma in the fusion flame fronts. This is also for preparation of specific experiments with PWPS lasers of sufficient contrast to explore the revolutionary new scheme. Figure 12 shows results of the ion density of the fusion flame when developing into solid density DT fuel after a PS laser pulse initiated the fusion flame. It is very interesting to see that the local ion density in the thin flame front moves with a velocity of $1.55 \times 10^{8} \mathrm{~cm} / \mathrm{s}$ and the density in the flame front is four times higher than the DT. This is an automatic result of the genuine two-fluid computation and agrees with the Rankine-Hugoniot theory of shock generation.

\section{Conclusions}

In conclusion, interaction of TW-PS laser pulses with plasma results in a skin layer mechanism for nonlinear (ponderomotive) force driven two-dimensional plasma blocks (pistons). This mechanism relies on a high contrast ratio for suppression of relativistic self-focusing. Space charge neutral plasma blocks are obtained with ion current densities larger than $10^{10} \mathrm{Amp} / \mathrm{cm}^{2}$. Using ions in the $\mathrm{MeV}$ range results in 1000 times higher proton or DT current densities than the proposed proton fast igniter requires. This should result in better conditions of this fast ignitor scheme. The ballistic focusing of the generated plasma blocks and then short time thermal expansion increases their thickness but keeps the high ion current densities. As shown here, this approach then provides conditions that are very favorable for efficient fast ignition of a fusion target. If successful, this approach to fast ignition could significantly simplify operation of an IFE plant, allowing very attractive energy production costs. What

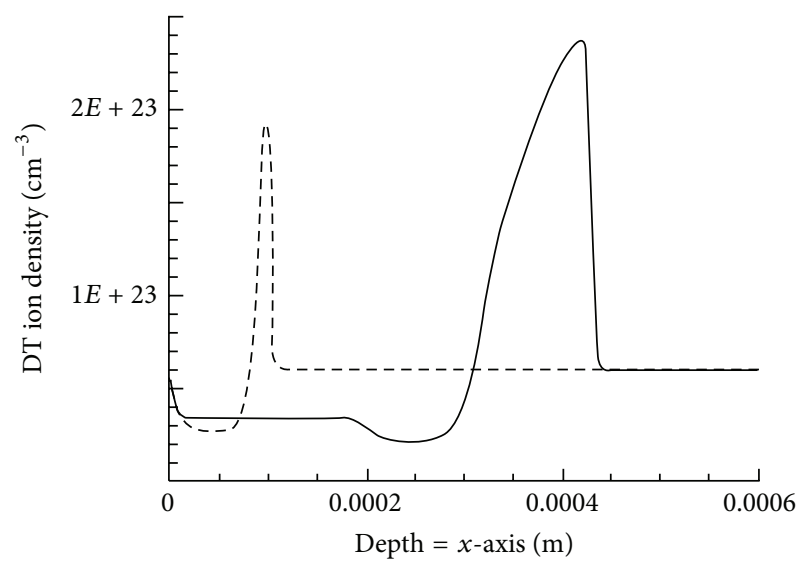

FIGURE 12: Genuine two fluid hydrodynamic computations [43, 44] of the ion density in solid DT after irradiation of a laser pulse of $10^{20} \mathrm{~W} / \mathrm{cm}^{2}$ of PS duration at the times 22 PS (dashed) and 225 PS after the initiation.

is evident is the measurement of the ultrahigh acceleration of plasmas at interaction with subpicosecond high intensity laser pulses if relativistic self-focusing is avoided by using very high contrast ratios for suppression of prepulses. The high contrast was initially necessary with $\mathrm{KrF}$ laser pulses to avoid amplified spontaneous emission (ASE). The generation of the ultrafast plane highly directed plasma blocks and avoiding relativistic self-focusing was confirmed also by $\mathrm{X}$ ray emission measurements [27] and the properties of the directed ion blocks based on the number of the fast ions limited to the dielectric strongly increased skin depth. [28]. The confirmation of the acceleration by the nonlinear (generalized ponderomotive) force in agreement with computed plane geometry conditions, Figure 1, [2, 25] demonstrated a nonthermal process where the laser energy was nearly completely converted into macroscopic acceleration of the space-charge neutral electron cloud in the plasma and the electrostatically attached ion cloud. These results are basically confirmed but may need more information about detailed properties. The use of the ultrafast plasma blocks for sideon ignition of uncompressed (or modestly compressed) solid fusion fuel following the early computations of Chu (1971) are based on hydrodynamic theory leading to ignition of high gain fusion with next available high contrast laser pulses of dozens of petawatt-picosecond laser pulses for DT fuel but even for HB11 fuel where less radioactivity is generated than from burning coal per gained energy (Hora et al. 2009) [51]. One problem is whether the hydrodynamic treatment for fusion is sufficient and whether not details of particle interpenetration [53] need to be included as known from Particle In Cell (PIC) computations as discovered by Wilks et al. (1991). A comparison between results from hydrodynamics and PIC evaluations for the range of interesting laser intensities (Limpouch et al. 2006) showed a rather good agreement. Laser generation at thin foils with high contrast ratio was studied in a number of experiments (see, e.g., [22]) and 
may need to be reconsidered as in similar cases [29] where the interaction by thermal processes need a comparison with nonlinear force effects.

\section{Conflict of Interests}

The authors declare that there is no conflict of interests regarding the publication of this paper.

\section{References}

[1] H. Hora, J. Badziak, F. P. Boody et al., "Effects of ps and ns laser pulses for giant ion source," Optics Communications, vol. 207, no. 1-6, pp. 333-338, 2002.

[2] H. Hora, J. Badziak, M. N. Read et al., "Fast ignition by laser driven particle beams of very high intensity," Physics of Plasmas, vol. 14, no. 7, Article ID 072701, 2007.

[3] M. Tabak, J. Hammer, M. E. Glinsky et al., "Ignition and high gain with ultrapowerful lasers," Physics of Plasmas, vol. 1, no. 5, pp. 1626-1634, 1994.

[4] E. M. Campbell, High Intensity Laser-Plasma Interaction and Applications to Inertial Fusion and High Energy Density Physics [Doctor of Science thesis], University of Western Sydney, New South Wales, Australia, 2005.

[5] C. Yamanaka and S. Nakai, "Thermonuclear neutron yield of $10^{12}$ achieved with Gekko XII green laser," Nature, vol. 319, no. 6056, pp. 757-759, 1986.

[6] E. Storm, "Press Conference Lawrence Livermore National Laboraotory," 1986.

[7] E. Storm, J. D. Lindl, E. M. Campbell et al., "Progress in laboratory high-gain ICF: prgress for the future Livermore," LLNL Report 47312, 1988.

[8] H. Hora, H. Azechi, Y. Kitagawa et al., "Measured laser fusion gains reproduced by self-similar volume compression and volume ignition for NIF conditions," Journal of Plasma Physics, vol. 60, no. 4, pp. 743-760, 1998.

[9] H. Hora and P. S. Ray, "Increased nuclear fusion yields of inertially confined DT plasma due to reheat," Zeitschrift Für Naturforschung A, vol. 33, pp. 890-894, 1978.

[10] R. C. Kirkpatrick and J. A. Wheeler, "Physics of DT ignition in small fusion targets," Nuclear Fusion, vol. 21, no. 3, pp. 389-401, 1981.

[11] H. Azechi, T. Jitsuno, T. Kanabe et al., "High-density compression experiments at ILE, Osaka," Laser and Particle Beams, vol. 9, no. 2, pp. 193-207, 1991.

[12] Y. Kato, K. Mima, N. Miyanaga et al., "Random phasing of high-power lasers for uniform target acceleration and plasmainstability suppression," Physical Review Letters, vol. 53, no. 11, pp. 1057-1060, 1984.

[13] H. Hora, "Smoothing and stochastic pulsation at high power laser-plasma interaction," Laser and Particle Beams, vol. 24, no. 3, pp. 455-463, 2006.

[14] J. M. Soures, R. L. Mccrory, C. P. Vernon et al., "Direct-drive laser-fusion experiments with the OMEGA, 60-beam, $>40 \mathrm{~kJ}$, ultraviolet laser system," Physics of Plasmas, vol. 3, pp. 2108-2112, 1996.

[15] J. D. Lindl, “The Edward Teller medal lecture: the evolution toward indirect drive and two decades of progress toward ignition and burn," in Edward Teller Lectures: Laser and Inertial Fusion Energy, H. Hora and G. H. Miley, Eds., pp. 121-147, Imperial College Press, London, UK, 2005.
[16] S. H. Glenzer, E. Moses, B. J. MacGowan et al., "Demonstration of ignition radiation temperatures in indirect-drive inertial confinement fusion hohlraums," Physical Review Letters, vol. 106, Article ID 085004, 5 pages, 2011.

[17] D. Strickland and G. Mourou, "Compression of amplified chirped optical pulses," Optics Communications, vol. 56, no. 3, pp. 219-221, 1985.

[18] G. Mourou and T. Tajima, "Ultraintense lasers and their applications," in Inertial Fusion Science and Applications, V. R. Tanaka, D. D. Meyerhofer, and J. Meyer-ter-Vehn, Eds., pp. 831839, Elsevier, Paris, France, 2002.

[19] S. Szatmari and F. P. Schäfer, "Simplified laser system for the generation of $60 \mathrm{fs}$ pulses at $248 \mathrm{~nm}$," Optics Communications, vol. 68 , pp. 196-201, 1988.

[20] S. Szatmari, "High-brightness ultraviolet excimer lasers," Applied Physics B, vol. 58, pp. 211-223, 1994.

[21] R. Sauerbrey, "Acceleration in femtosecond laser-produced plasmas," Physics of Plasmas, vol. 3, no. 12, pp. 4712-4716, 1996.

[22] M. Kaluza, J. Schreiber, M. I. K. Santala et al., "Influence of the laser prepulse on proton acceleration in thin-foil experiments," Physical Review Letters, vol. 93, no. 4, Article ID 045003, 2004.

[23] H. Hora, Plasemas at High Temperature and Density, Springer, Heidelberg, Germany, 1991.

[24] F. F. Chen, "Physical mechanisms for laser-plasma parametric instabilities," in Laser Interaction and Related Plasma Phenomena, H. J. Schwarz and H. Hora, Eds., vol. 3, pp. 291-313, Plenum Press, New York, NY, USA, 1974.

[25] H. Hora, Physics of Laser Driven Plasmas, John Wiley \& Sons, New York, NY, USA, 1981.

[26] H. Hora, "Theory of relativistic self-focuing of laser radiation in Plasmas," Journal of the Optical Society of America, vol. 65, no. 8, pp. 882-886, 1975.

[27] P. Zhang, J. T. He, D. B. Chen et al., "X-ray emission from ultraintense-ultrashort laser irradiation," Physical Review E, vol. 57, pp. 3746-3752, 1998.

[28] J. Badziak, A. A. Kozlov, J. Makowski et al., "Investigations of ion streams emitted from plasma produced with a high-power picosecond laser," Laser and Particle Beams, vol. 17, no. 2, pp. 323-329, 1999.

[29] H. Hora, "Skin-depth theory explaining anomalous picosecond-terawatt laser plasma interaction II," Czechoslovak Journal of Physics, vol. 53, no. 3, pp. 199-217, 2003.

[30] T. E. Cowan, M. D. Perry, M. H. Key et al., "High energy electrons, nuclear phenomena and heating in petawatt lasersolid experiments," Laser and Particle Beams, vol. 17, no. 4, pp. 773-783, 1999.

[31] L. Cicchitelli, H. Hora, and R. Postle, "Longitudinal field components for laser beams in vacuum," Physical Review A, vol. 41, no. 7, pp. 3727-3732, 1990.

[32] T. Häuser, W. Scheid, and H. Hora, "Theory of ions emitted from a plasma by relativistic self-focusing of laser beams," Physical Review A, vol. 45, no. 2, pp. 1278-1281, 1992.

[33] X. Yang, G. H. Miley, K. A. Flippo, and H. Hora, "Energy enhancement for deuteron beam fast ignition of a precompressed inertial confinement fusion target," Physics of Plasmas, vol. 18, no. 3, Article ID 032703, 2011.

[34] J. Badziak, S. Głowacz, S. Jablłoński et al., "Production of ultrahigh ion current densities at skin-layer subrelativistic laserplasma interaction," Plasma Physics and Controlled Fusion, vol. 46, no. 12, pp. B541-B555, 2004. 
[35] J. Badziak, S. GŁowacz, S. JabŁoński, P. Parys, J. WoŁowski, and H. Hora, "Generation of picosecond high-density ion fluxes by skin-layer laser-plasma interaction," Laser and Particle Beams, vol. 23, no. 2, pp. 143-147, 2005.

[36] K. Flippo, privatecommunication, 2011.

[37] I. B. Földes, J. S. Bakos, K. Gál et al., "Properties of high harmonics generated by ultrashort UV laser pulses on solid surfaces," Laser Physics, vol. 10, no. 1, pp. 264-269, 2000.

[38] G. Veres, G. Kocsis, E. Rácz, and S. Szatmári, "Doppler shift of femtosecond laser pulses from solid density plasmas," Applied Physics B: Lasers and Optics, vol. 78, no. 5, pp. 635-638, 2004.

[39] I. B. Földes and S. Szatmári, "On the use of KrF lasers for fast ignition," Laser and Particle Beams, vol. 26, no. 4, pp. 575-582, 2008.

[40] A. Schlüter, "Dynamik des plasmas-I: grundgleichungen, plasma in gekreutzten feldern," Zeitschrift für Naturforschung A, vol. 5, pp. 72-78, 1950.

[41] H. Alfven, Cosmic Plasma Dordrecht, Reidel, 1981.

[42] R. Kulsrud, "Book review: Hannes Alfven," Physics Today, vol. 34, no. 4, p. 56, 1983.

[43] P. Lalousis and H. Hora, "First direct electron and ion fluid computation of high electrostatic fields in dense inhomogeneous plasmas with subsequent nonlinear laser interaction," Laser and Particle Beams, vol. 1, no. 3, pp. 283-304, 1983.

[44] H. Hora, P. Lalousis, and S. Eliezer, "Analysis of the inverted double layers produced by nonlinear forces in a laser-produced plasma," Physical Review Letters, vol. 53, no. 17, pp. 1650-1652, 1984.

[45] S. Głowacz, J. Badziak, S. Jabłonski, and H. Hora, "Numerical modelling of production of ultrahigh-current-density ion beams by short-pulse laser-plasma interaction," Czechoslovak Journal of Physics, vol. 54, no. 3, pp. C460-C467, 2009.

[46] S. Glowacz, H. Hora, J. Badziak, S. Jablonski, Y. Cang, and F. Osman, "Analytical description of rippling effect and ion acceleration in plasma produced by a short laser pulse," Laser and Particle Beams, vol. 24, no. 1, pp. 15-25, 2006.

[47] R. Sadighi-Bonabi, E. Yazdani, Y. Cang, and H. Hora, "Dielectric magnifying of plasma blocks by nonlinear force acceleration with delayed electron heating," Physics of Plasmas, vol. 17, no. 11, Article ID 113108, 5 pages, 2010.

[48] J. L. Bobin, "Nuclear fusion reactions in fronts propagating in solid DT," in Laser Interaction and Related Plasma Phenomena, H. Schwarz and H. Hora, Eds., vol. 4, pp. 465-494, Plenum Press, New York, NY, USA, 1974.

[49] J. L. Nuckolls and L. Woods, "Future of inertial fusion energy," in Proceedings of the International Conference on Nuclear Energy Systems ICNES Albuquerque, H. S. Park and B. Remington, Eds., 2002, Astrophyhsics and Space Science.

[50] H. Hora, B. Malekynia, M. Ghoranneviss, G. H. Miley, and X. $\mathrm{He}$, "Twenty times lower ignition threshold for laser driven fusion using collective effects and the inhibition factor," Applied Physics Letters, vol. 93, no. 1, Article ID 011101, 3 pages, 2008.

[51] H. Hora, G. H. Miley, M. Ghoranneviss, B. Malekynia, N. Azizi, and X.-T. He, "Fusion energy without radioactivity: laser ignition of solid hydrogen-boron (11) fuel," Energy and Environmental Science, vol. 3, no. 4, pp. 479-486, 2010.

[52] Y. Li, "Nuclear power without radioactivity," in Highlights in Chemical Technology, vol. 7, Royal Chemical Society, London, UK, 2010.

[53] H. Hora, "Interpenetration burn for controlled inertial confinement fusion by nonlinear forces," Atomkernenergie, Kerntechnik, vol. 42, no. 1, pp. 7-10, 1983. 


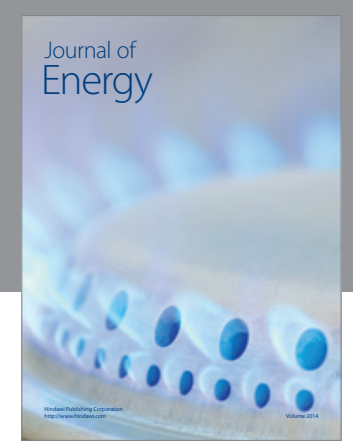

Journal of

Industrial Engineering
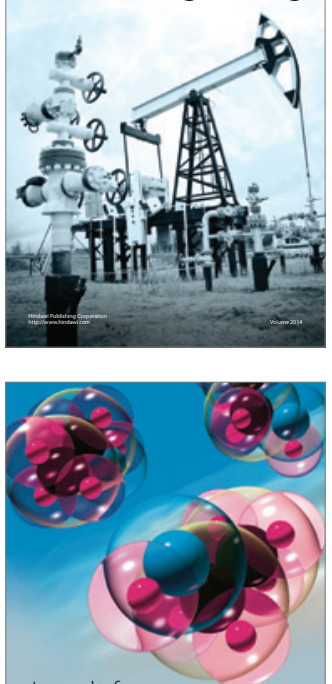

Fuels
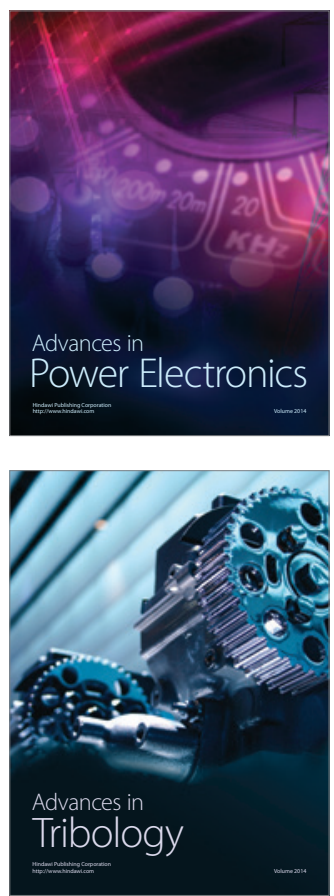

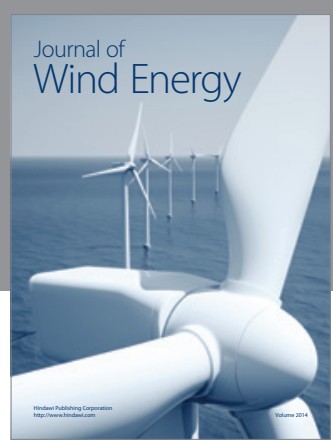

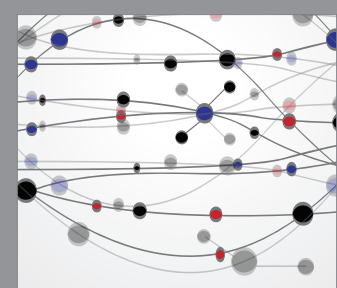

The Scientific World Journal

Submit your manuscripts at http://www.hindawi.com

Journal of

Structures
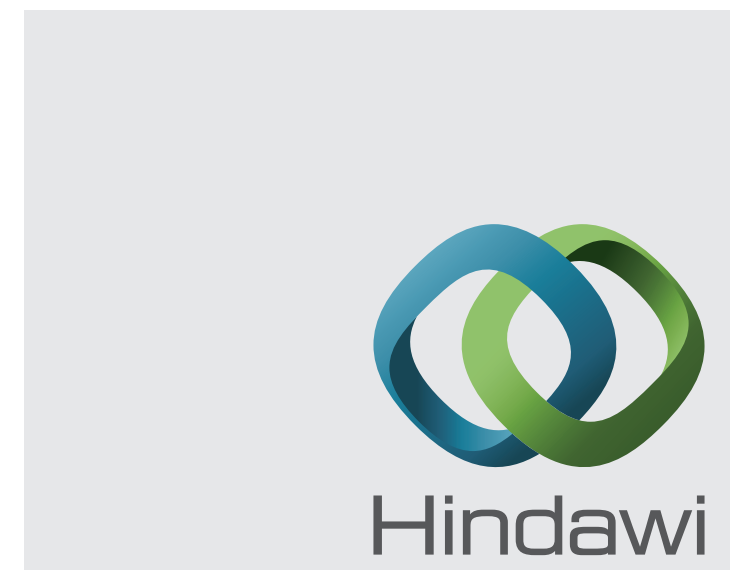

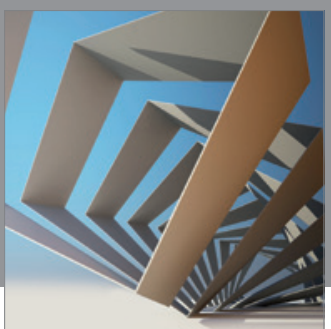

Rotating

Machinery
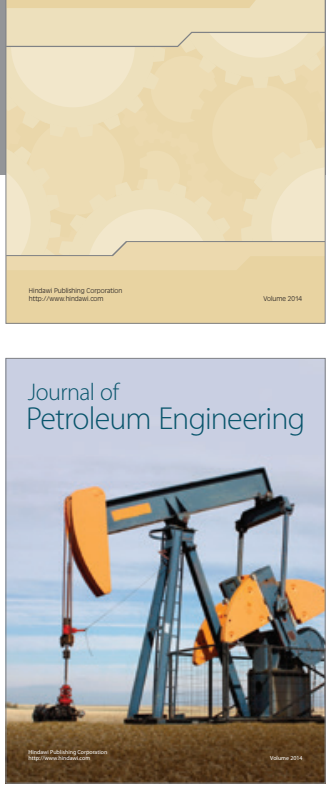

Journal of

Solar Energy
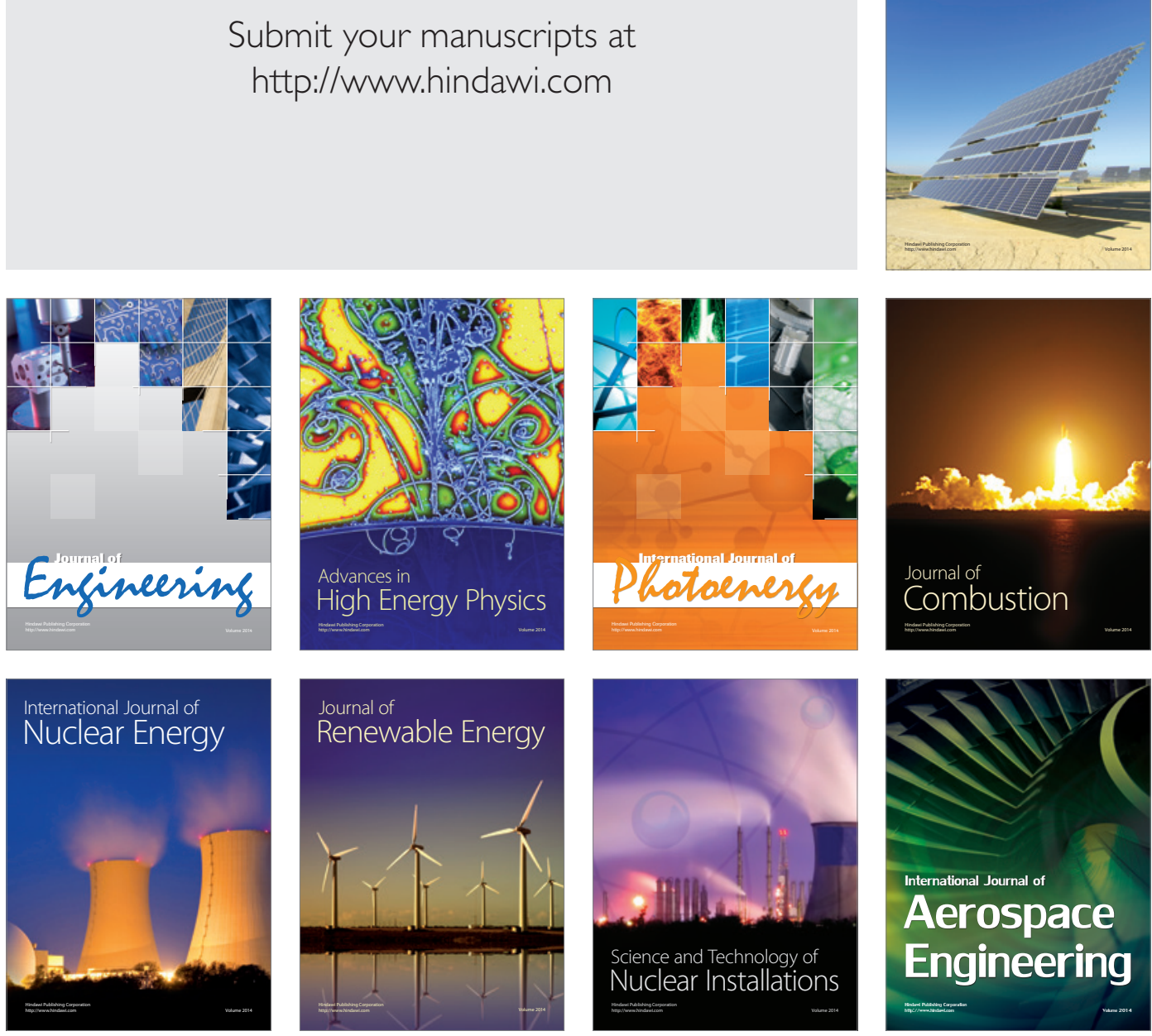